\title{
Techniques for Input Ripple Current Cancellation: Classification and Implementation
}

\author{
N. K. Poon, Member, IEEE, J. C. P. Liu, Member, IEEE, C. K. Tse, Senior Member, IEEE, and \\ M. H. Pong, Senior Member, IEEE
}

\begin{abstract}
Switching power supplies, due to their high-frequency switching operation, draw a substantial amount of input ripple current. Such ripple current manifests itself as electromagnetic interference (EMI). Active and passive ripple cancellation methods have been proposed in the past. This paper discusses the cancellation methods, and classifies the basic circuit configurations for ripple current cancellation. The basis of discussion is a "nullification" process, which can be described effectively in terms of the ideal circuit element nullor. The initial focus is on differential-mode noise cancellation, but the basic technique can be applied equally to cancelling common-mode noise. The classification provides a clear guideline for synthesizing practical cancellation circuits. Both active and passive implementation examples are given and experimentally demonstrated.
\end{abstract}

Index Terms-Electromagnetic interference, ripple current cancellation, switching regulators.

\section{INTRODUCTION}

I NPUT ripple current from a switching power supply contains considerable low-order harmonics, necessitating extra design efforts for reducing or limiting the amount of electromagnetic interference (EMI) in the frequency range concerned. To achieve electromagnetic compatibility (EMC) compliance, filtering approaches are commonly used. Such approaches usually involve bulky components so as to provide effective suppression of the relatively high content of low-order harmonics [1], [2]. The use of bulky filters, however, does not seem to be an economical design solution for the light and shrinking switching power supplies.

Active and passive ripple cancellations provide alternative approaches to the problem. Some practical forms of active cancellation circuits have been reported recently [3]-[11]. Although the basic method has been known for some time, there seems to be no systematic synthesis procedure of active or passive noise cancelling circuits that can expedite the practical design of such circuits. In this paper, we attempt to generalize the approach and derive a family of basic circuit configurations which can be implemented in both active and passive forms. We will describe the

Manuscript received January 4, 2000; revised May 22, 2000. Recommended by Associate Editor N. Femia.

N. K. Poon and J. C. P. Liu are with the Department of Electrical and Electronic Engineering, University of Hong Kong, Hong Kong, and also with the Department of Electronic and Information Engineering, Hong Kong Polytechnic University, Hong Kong (e-mail: nkpoon@eee.hku.hk; cpliu@eee.hku.hk).

C. K. Tse is with the Department of Electronic and Information Engineering, Hong Kong Polytechnic University, Hong Kong (e-mail: cktse@eie.polyu.edu.hk).

M. H. Pong is with the Department of Electrical and Electronic Engineering,

University of Hong Kong, Hong Kong (e-mail: mhp@eee.hku.hk).

Publisher Item Identifier S 0885-8993(00)09801-X. basic theory in terms of the ideal circuit element, nullor, which is composed of a nullator and a norator. The idea is to "null" the ripple current by placing a nullator at a suitable position in the circuit. The norator is then included to supply current or voltage at some other location, as would be necessary to restore the normal loading or input condition. In fact, many previously reported active filters and noise cancelling circuits can be synthesized from these basic configurations [3]-[11]. Also, the technique is equally applicable to combating common-mode noise, although our discussion focuses on differential-mode noise for simplicity. We will present a comparative study of the basic approaches, and discuss their practical implementations. Finally, results from experimental tests will be presented for verification.

\section{Possibilities OF ZERo INPUT RipPle CURRENT}

The ideal circuit element nullator is a two-terminal device whose voltage and current are zero, while the norator is one whose voltage and current can assume any value. Fig. 1(a) shows their circuit symbols. Obviously any solvable circuit must have equal numbers of nullators and norators. An ideal op-amp, for example, can be represented by a pair of nullator and norator [12].

Consider now a switching power supply which draws an ac ripple current from the mains. Let $Z_{\text {in }}$ be the mains impedance and $i_{s}$ be the equivalent ac current that is being injected to the mains. Suppose an input capacitor, $C_{\mathrm{in}}$, is connected across the mains. Fig. 1(b) shows the ac equivalent circuit model. If we wish to null off the current that flows into $Z_{\text {in }}$, we can either connect a nullator in series or in parallel with $Z_{\text {in }}$. Four configurations are possible, as shown in Fig. 1(c) through (d). We denote, for brevity, these configurations as Types I, II, III, and IV.

In the configurations of Types I and II, a nullator is in series with the mains impedance. Zero current thus flows in $Z_{\mathrm{in}}$. However, such an arrangement will necessitate the presence of a norator in order to satisfy Kirchhoff's laws. Likewise, for Types III and IV, a nullator is in parallel with the mains impedance, and a norator is again mandatory for fulfilling Kirchhoff's laws. Specifically, the norator in each case must take up appropriate voltage or current. For the circuits of Types I and III, we have

$$
\left\{\begin{array}{l}
v_{n}=-v_{C} \\
i_{n}=0
\end{array}\right.
$$

and for the circuits of Types II and IV, we have

$$
\left\{\begin{array}{l}
v_{n}=0 \\
i_{n}=i_{s}
\end{array} .\right.
$$




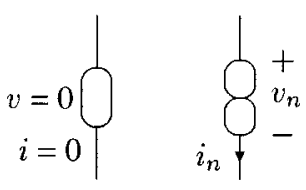

(a)

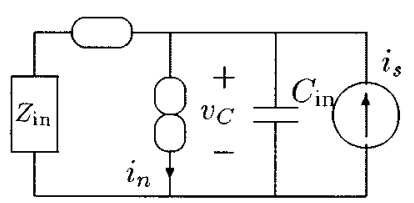

(d)

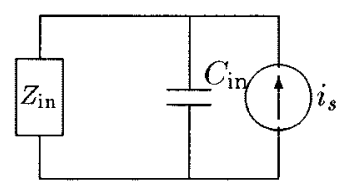

(b)

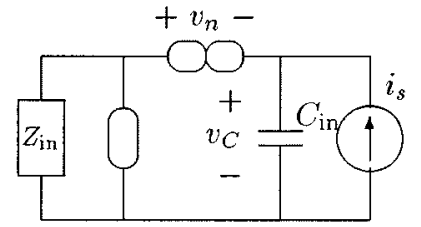

(e)

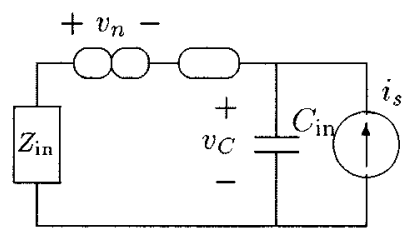

(c)

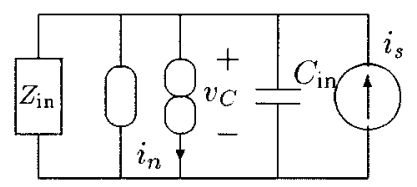

(f)

Fig. 1. (a) Nullor symbols, (b) simplified ac equivalent model for input current and mains interaction and (c)-(f) ideal circuits for nulling input ripple current. (c) Type I. (d) Type II. (e) Type III. (f) Type IV.

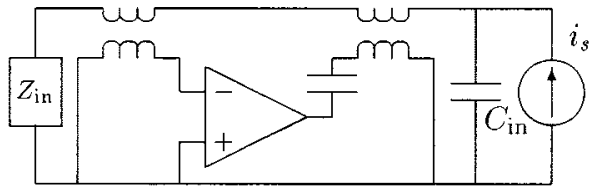

(a)

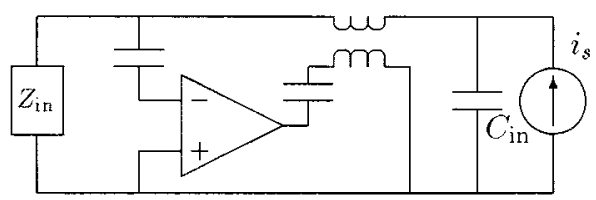

(c)

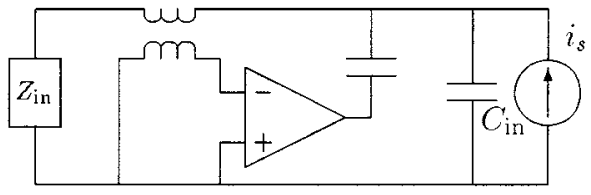

(b)

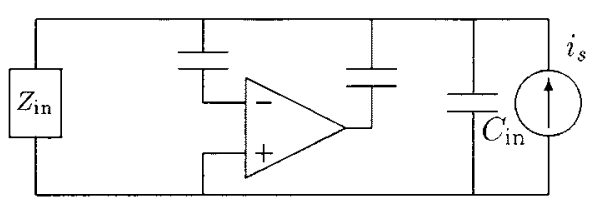

(d)

Fig. 2. Active circuit realization of input ripple current nullifier: (a) corresponding to Type I [Fig. 1(c)], (b) corresponding to Type II [Fig. 1(d)], (c) corresponding to Type III [Fig. 1(e)], and (d) corresponding to Type IV [Fig. 1(f)].

Note that, in all cases, the norator has either zero voltage or current. Thus, the power dissipation of the nullor, in each case, is theoretically zero.

It should be obvious that configurations of Types I and III would mandatorily require the presence of a decoupling capacitor $C_{\text {in }}$, whereas those of Types II and IV can omit such a capacitor theoretically.

\section{ACTIVE CIRCUIT REALIZATION}

\section{A. Basic Configurations of Active Nullifiers}

The conceptual circuits derived in the foregoing section can be realized using active devices. Specifically, nullors are implementable by active devices like op-amps and transistors [12]. Whenever necessary, isolation transformers and dc level shifters may be included. Moreover, realization is not unique, and there can be many possible realization styles. Fig. 2 shows the circuit implementations for the configurations shown in Fig. 1(c)-(f). These circuits use op-amps as nullors, and transformers for voltage level alignments.

In theory the resultant input current would be reduced to zero if the op-amp was ideal. However, in practice, the gain-bandwidth product of the op-amp and the sensing circuit bandwidth limit the performance. An approximation for the noise suppression performance can be represented by

$$
\frac{V_{N}}{V_{N}^{\prime}}=\frac{f_{n}}{f_{\beta}}
$$

where

$V_{N} \quad$ noise voltage across $Z_{\text {in }}$ if no nullifier circuit is used;

$V_{N}^{\prime} \quad$ noise voltage across $Z_{\text {in }}$ when a nullifier circuit with an open-loop gain-bandwidth product of $f_{\beta}$ is used;

$f_{n} \quad$ noise bandwidth.

Note that if $f_{\beta}=\infty$, we have perfect nullification.

Fortunately, the bandwidth of the op-amp does not need to cover the whole EMI spectrum for most practical purposes since the noise current spectrum generally falls off at $40 \mathrm{~dB} / \mathrm{dec}$ at high frequencies. Most existing designs of differential-mode filters focus on the first several harmonics of the switching frequency. Hence, it usually suffices to choose an op-amp whose gain-bandwidth product is high enough to ensure good nulling performance for the first several harmonics of the switching frequency. 


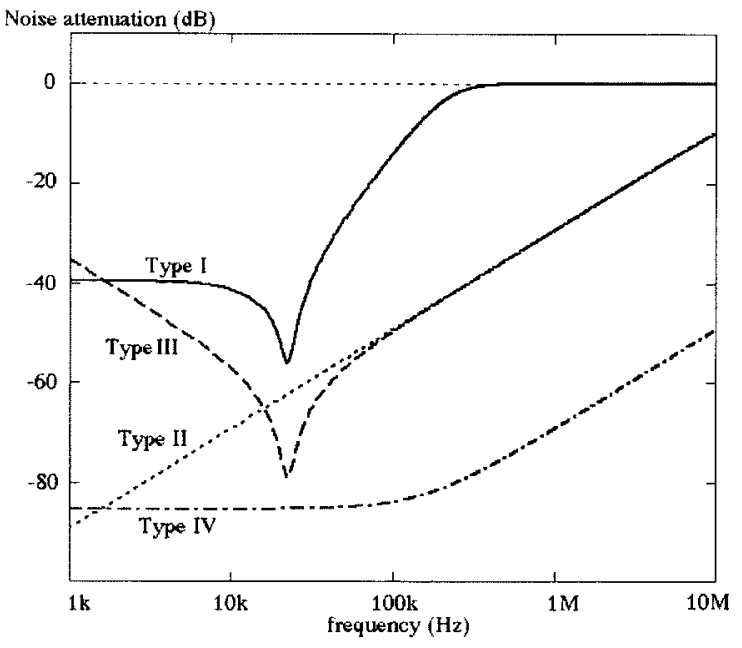

(a)

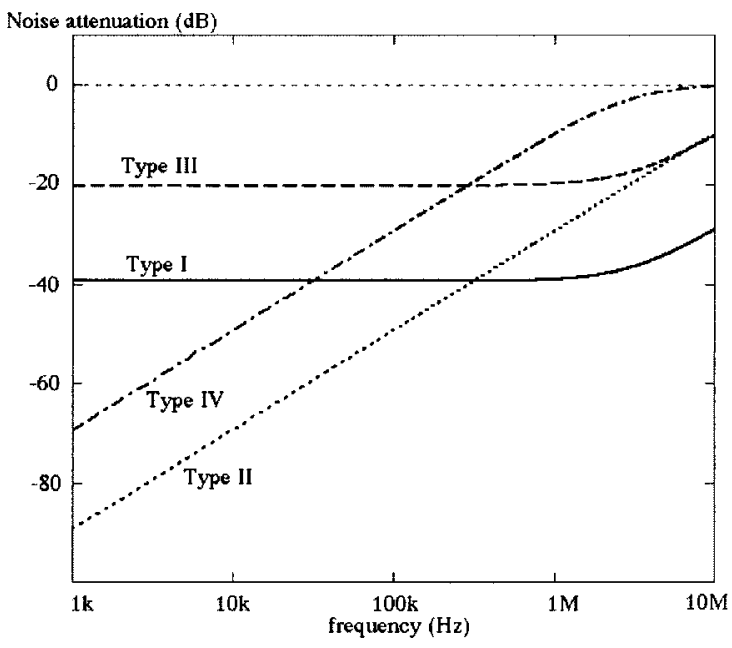

(b)

Fig. 3. Comparison of noise attenuation of the four types of active nullifiers for (a) $Z_{i n}=100 \Omega \| 100 \mu \mathrm{H}$ and (b) $Z_{i n}=0.1 \Omega$. Assume openloop gains for all types are $2 \times 10^{6} /(1+j f / 15)$.

TABLE I

PROPERTIES OF ACTIVE NULLIFIERS

\begin{tabular}{c|ccc}
\hline Type & Amplifier type & Amplifier gain & Noise attenuation \\
\hline I & transresistance & $z_{t}$ & $\frac{1+\frac{1}{s C_{\text {in }} Z_{\text {in }}}}{1+\frac{z_{t}}{Z_{\text {in }}}+\frac{1}{s C_{\text {in }} Z_{\text {in }}}}$ \\
II & current gain & $A_{i}$ & $\frac{1}{1+A_{i}}$ \\
III & voltage gain & $A_{v}$ & $\frac{1+\frac{1}{s C_{\text {in }} Z_{\text {in }}}}{1+A_{v}+\frac{1}{s C_{\text {in }} Z_{\text {in }}}}$ \\
IV & transconductance & $g_{t}$ & $\frac{1}{1+g_{t} Z_{\text {in }}}$ \\
\hline
\end{tabular}

\section{B. Comparison of the Basic Active Nullifiers}

Although all basic nullifier circuits in theory achieve the same function of removing the input ripple current, they do have relative advantages and disadvantages when considered from a practical point of view. In this section, we attempt to provide a comparative review of the basic configurations, the aim being to identify their inherent properties that make them particularly suitable or unsuitable for practical applications.

To compare the effectiveness of the nullifier circuits, we calculate for each case the noise attenuation, which is defined as

$$
\mathrm{NA}=\frac{\left[I_{r}\right]_{\text {with nullifier }}}{\left[I_{r}\right]_{\text {without nullifier }}}
$$

where $\left[I_{r}\right]_{\text {with nullifier }}$ and $\left[I_{r}\right]_{\text {without nullifier }}$ are the input ripple current with and without the use of a nullifier circuit, respectively.

For the active nullifier of Type I [Fig. 2(a)], the noise attenuation is readily found as

$$
\mathrm{NA}_{\mathrm{I}}=\frac{1+\frac{1}{s C_{\mathrm{in}} Z_{\mathrm{in}}}}{1+\frac{z_{t}}{Z_{\mathrm{in}}}+\frac{1}{s C_{\mathrm{in}} Z_{\mathrm{in}}}}
$$

where $z_{t}$ is the open-loop trans-impedance gain from the sensed input current to the counteracting voltage, as shown

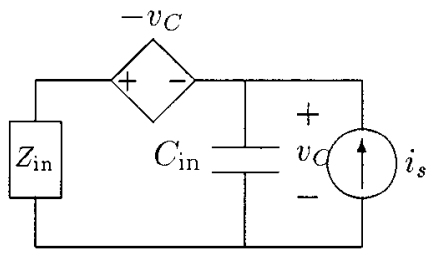

(a)

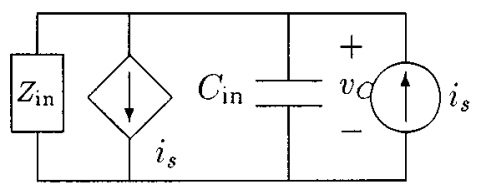

(b)

Fig. 4. Alternative implementations for (a) circuits of Types I and III and (b) circuits of Types II and IV. $C_{\text {in }}$ can be omitted in (b).

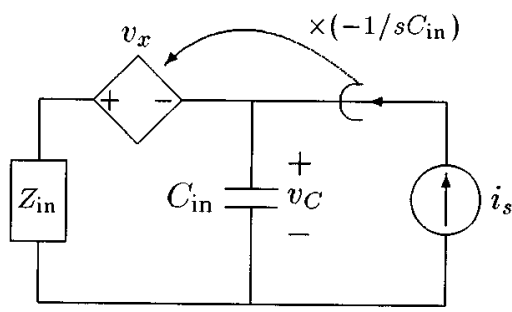

(a)

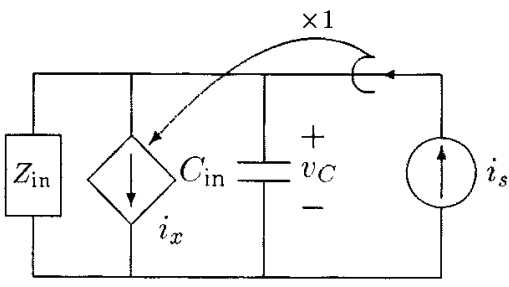

(b)

Fig. 5. Conceptual circuit realization of input ripple current nullifier by feedforward of noise current: (a) passive type I corresponding to Fig. 4(a) and (b) passive type II corresponding to Fig. 4(b). $C_{\text {in }}$ can be omitted in (b). 
in Fig. 2(a). Note that the presence of $C_{\text {in }}$ is necessary for absorbing the ripple current. However, for the active nullifier of Type II [Fig. 2(b)], the noise attenuation is found as

$$
\mathrm{NA}_{\mathrm{II}}=\frac{1}{1+A_{i}}
$$

where $A_{i}$ is the open-loop current gain from the sensed input current to the counteracting current, as shown in Fig. 2(b). Clearly, $C_{\text {in }}$ is not mandatory in this case. Moreover, for the active nullifier of Type III [Fig. 2(c)], the noise attenuation is

$$
\mathrm{NA}_{\mathrm{III}}=\frac{1+\frac{1}{s C_{\mathrm{in}} Z_{\mathrm{in}}}}{1+A_{v}+\frac{1}{s C_{\mathrm{in}} Z_{\mathrm{in}}}}
$$

where $A_{v}$ is the open-loop voltage gain from the sensed input voltage to the counteracting voltage, as shown in Fig. 2(c). Note that $C_{\mathrm{in}}$ is mandatory for this type of active nullifier. Finally, for the active nullifier of Type IV [Fig. 2(d)], the noise attenuation is

$$
\mathrm{NA}_{\mathrm{IV}}=\frac{1}{1+g_{t} Z_{\mathrm{in}}}
$$

where $g_{t}$ is the open-loop trans-conductance gain from the sensed input voltage to the counteracting current, as shown in Fig. 2(d). For this one, $C_{\text {in }}$ is not necessary.

From the above-derived expressions, one can conclude that the effectiveness of the methods depend on the size of $Z_{\text {in }}$ (pick-up impedance), the size of the shunt capacitance if exists, and the open-loop gain of the active device used. Specifically, the following observations are made.

1) Types I and III nullifiers require the use of decoupling capacitance, while Types II and IV do not.

2) Active nullifiers of Types I and III have identical performance if $z_{t} / Z_{\text {in }}=A_{v}$.

3) Active nullifiers of Types II and IV have identical performance if $A_{i}=g_{t} Z_{\text {in }}$.

4) For $Z_{\text {in }} \gg z_{t} / A_{v}$, active nullifiers of Type I perform better than those of Type III, and vice versa.

5) For $Z_{\text {in }} \gg A_{i} / g_{t}$, active nullifiers of Type II perform better than those of Type IV, and vice versa.

6) When $Z_{\text {in }} \gg 1 / s C_{\text {in }}$, active nullifiers of Types II and III have similar performance. Hence, at high frequencies, their performances are asymptotically the same.

A convenient way to compare these nullifiers is to plot the noise attenuation for different $Z_{\text {in }}$. Fig. 3(a) and (b) compare the noise attenuation of the four active nullifiers for two different $Z_{\text {in }}$ 's. In all cases, we assume that $C_{\mathrm{in}}=1 \mu \mathrm{F}$, and the active device has an open-loop gain of $2 \times 10^{6}$ and a low-frequency roll-off at $15 \mathrm{~Hz}$, i.e., $z_{t}=A_{v}=A_{i}=g_{t}=2 \times 10^{6} /(1+j f / 15)$. Table I summarizes the essential properties.

\section{PASSIVE Circuit Realization}

The foregoing has illustrated the use of a nullator to force the input ripple current to zero, with a norator helping to keep the null condition by supplying a suitable voltage or current. Taking

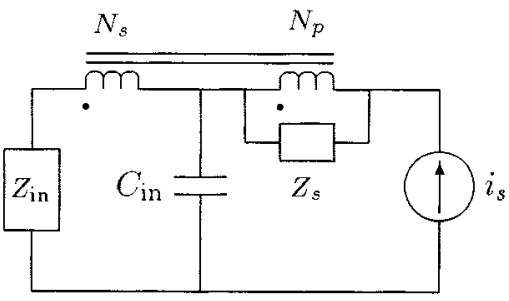

(a)

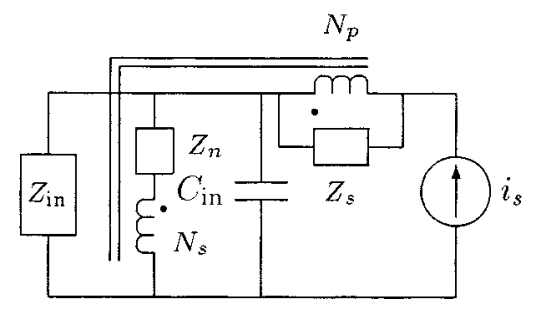

(b)

Fig. 6. Passive circuit realization of input ripple current nullifier based on feedforward of noise current: (a) passive type I corresponding to Fig. 5(a) and (b) passive type II corresponding to Fig. 5(b). $C_{\text {in }}$ can be omitted in (b).

TABLE II

EXAMPLES OF REPORTED CIRCUITS

\begin{tabular}{c|c|c}
\hline Previous work & Type & Mode \\
\hline Farkas-Schlecht [3] & active II & differential \\
Vlatkovic et al. [4] & active I & differential \\
Lee-Cho [5] & active I & differential \\
Hamill [6] & active II & differential \\
Takahashi et al. [8] & active II & common \\
Julian et al. [9] & passive I & common \\
Ogasawara et al. [10] & active III & common \\
Hamill-Krein [11] & passive II & differential \\
\hline
\end{tabular}

a closer look, the null condition can in fact be maintained if a voltage or current source of the right magnitude is applied in lieu of the norator. Two cases can be identified according to Fig. 1 .

1) For the circuits of Types I and III, the norator needs to supply a voltage of $-v_{C}$ according to (1) in order to maintain the null condition.

2) For the circuits of Types II and IV, the norator needs to supply a current of $i_{s}$ according to (2) in order to maintain the null condition.

Now it is apparent that if we can place a voltage (current) source that assumes exactly the value of $-v_{C}\left(i_{s}\right)$ in series (parallel) to the input, then we would be able to make the input current zero. Moreover, this approach may need no nullator and require only passive components. Fig. 4 illustrates these alternative configurations.

The circuits of Fig. 4 can be conceptually constructed by direct feedforward of the noise current, as illustrated in Fig. 5. A 


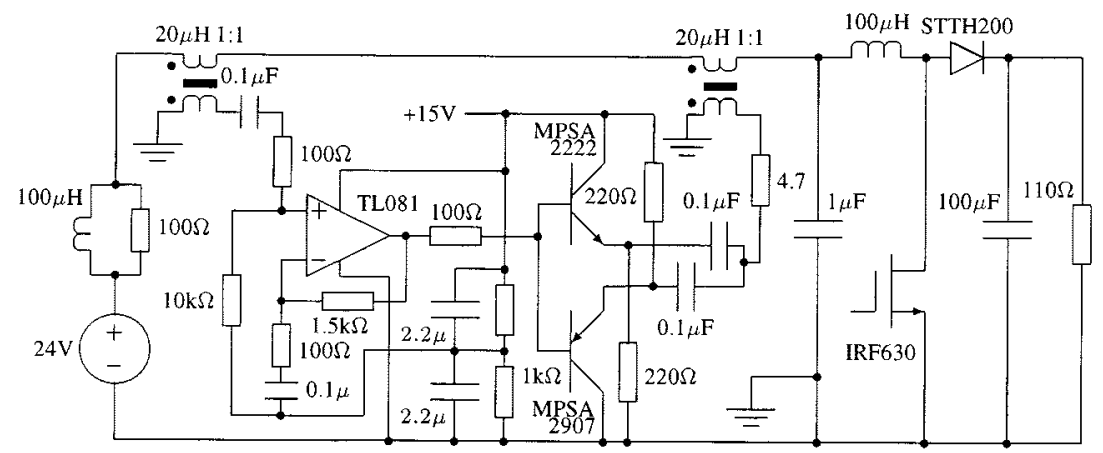

(a)

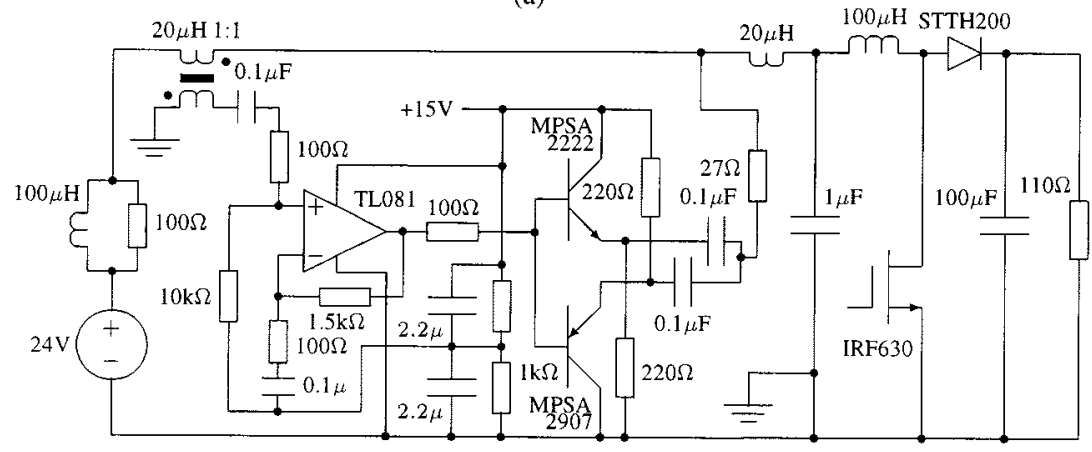

(b)

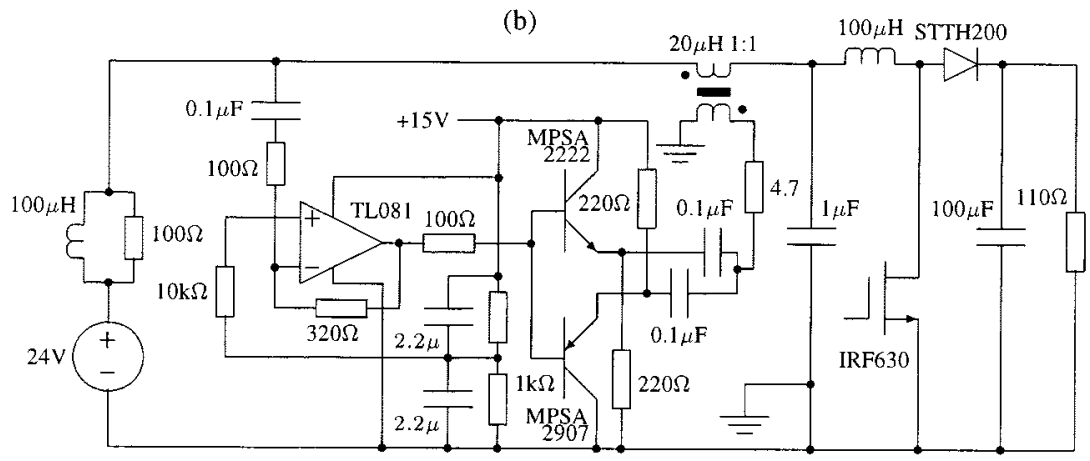

(c)

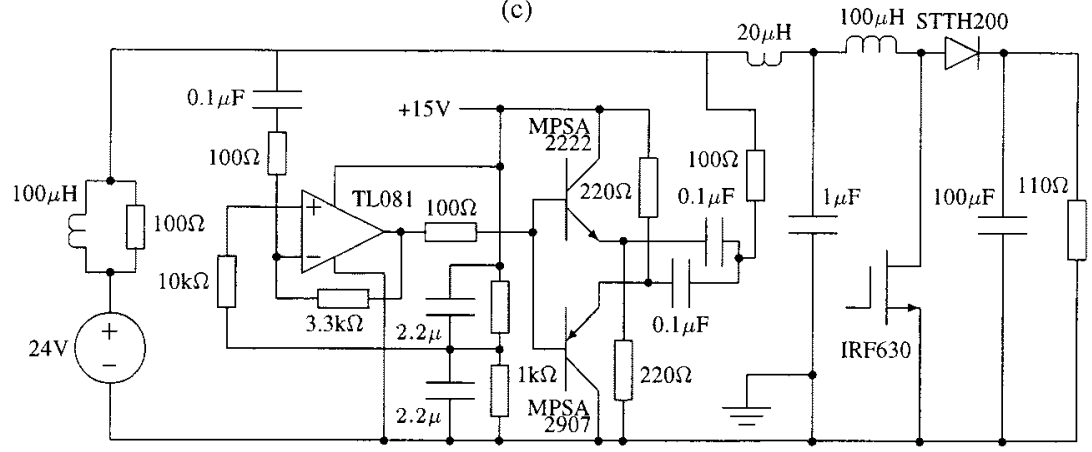

(d)

Fig. 7. Experimental circuits for active nullification. All $20 \mu \mathrm{H}$ transformers/inductors are FR $11 \times 5 \times 4$ iron power toroid. (a) Type I. (b) Type II. (c) Type III. (d) Type IV.

straightforward implementation of these feedforward circuits is to use a transformer, with suitable sensing impedance, as shown in Fig. 6. For the circuit of Fig. 6(a), nulling can be achieved if the transformer secondary produces a voltage which is very close to $v_{C}$, as explained earlier. This requires that the sensing impedance $Z_{s}$ be given by

$$
Z_{s}=\frac{s L_{T 1}}{s^{2} L_{T 1} C_{\mathrm{in}} n_{T}-1}
$$




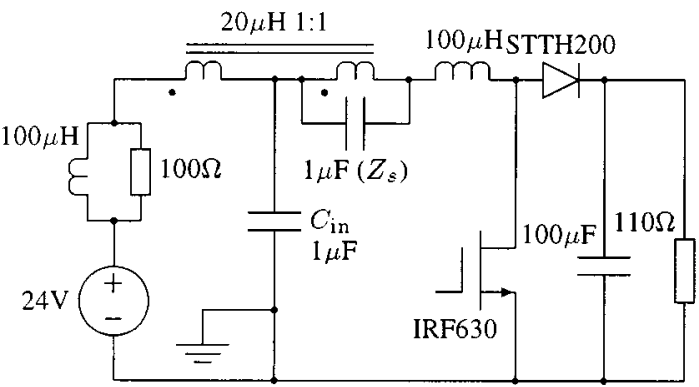

(a)

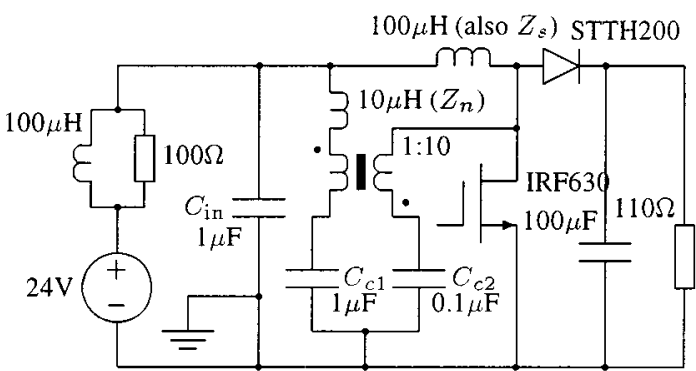

(b)

Fig. 8. Experimental circuits for passive nullification (a) corresponding to Fig. 6(a) and (b) corresponding to Fig. 6(b). All $20 \mu \mathrm{H}$ transformers/inductors are FR11 $\times 5 \times 4$ iron power toroid.

where $L_{T 1}$ is the magnetizing inductance seen from the transformer primary and $n_{T}$ is the turns ratio $N_{s} / N_{p}$. If $s L_{T 1} n_{T} \gg$ $1 / s C_{\text {in }}$, we have

$$
Z_{s} \approx \frac{1}{s C_{\text {in }} n_{T}}
$$

For the circuit of Fig. 6(b), nulling can be achieved if the transformer secondary produces a current which is very close to $i_{s}$. Ignoring the transformer leakage, the requirement can be expressed as

$$
Z_{n}=\frac{Z_{s} n_{T}\left(1-n_{T}\right)}{1+\frac{Z_{s}}{s L_{T 1}}}
$$

which can be approximated as $Z_{n} \approx Z_{s} n_{T}$ if $n_{T} \ll 1$ and $s L_{T 1} \gg Z_{s}$. Note that in this case, the decoupling capacitor $C_{\text {in }}$ can be omitted.

Remarks: Passive nulling has been proposed repeatedly by various authors, as surveyed by Hamill and Krein [11]. Our intention here, however, is to generalize the technique in terms of nullification of input current. Furthermore, it is possible to derive alternative realizations based on sensing voltage. For instance, referring to Fig. 6(a), the transformer primary may be connected across $C_{\text {in }}$, sensing the noise voltage, and the secondary produces the voltage source as required in the basic configuration shown in Fig. 5(a). Table II shows some previously reported circuits, including both active and passive approaches.

\section{EXPERIMENTAL VERIFICATION}

We have constructed four active nullifier circuits corresponding to the four basic types discussed in Section III, and also two passive nullifier circuits corresponding to the two basic types discussed in Section IV. The schematics of these six experimental circuits are shown in Figs. 7 and 8. All circuits except the one shown in Fig. 8(b) are obvious derivatives of the respective basic forms. Moreover, upon closer inspection, the circuit of Fig. 8(b) is indeed derivable from the basic circuit of Fig. 6(b). First, we make use of the inductance of the boost converter to serve as $Z_{s}$. Second, we shift one end of the transformer to the lower side of $C_{\text {in }}$ (i.e., ground). Such shifting can be done because $C_{\mathbf{i n}}$ is a virtual ac short-circuit under the nullification condition. The circuit of Fig. 8(b) is therefore equivalent to that of Fig. 6(b). Moreover, it is interesting to note that the coupled filter discussed in Hamill and Krein [11] is in fact directly derivable from the basic circuit of Fig. 6(b).

Each circuit is applied to a boost converter operating at 100 $\mathrm{kHz}$ and stepping up voltage from $24 \mathrm{~V}$ to $48 \mathrm{~V}$. For each case, we measure the input current waveform and its frequency spectrum. Results are shown in Figs. 9 and 10 for the active nullifiers and the passive nullifiers respectively. For comparison, in Fig. 9(b), we also give the measured input current waveforms and spectra when no nullifier is used and when only a $20 \mu \mathrm{H}$ choke is used. In all cases, we observe significant reduction in the input current ripple that can be gained using the nullification technique.

Remarks: Comparing the experimental results and the analysis presented earlier, one can observe some obvious discrepancies between the experimental measurements and the analytical predictions, notably in respect of the relative amount of noise attenuation of different types of active nullifiers. This, in fact, can be easily understood since our analysis assumes equal gain values for all $Z_{t}, A_{v}, A_{i}$, and $g_{t}$, while practical implementations of these gains are subject to varying limitations and requirements for maintaining loop stability under different implementations.

\section{CONCLUSION}

Noise cancellation techniques are useful in power electronics for control of EMI. Literature abounds with practical circuits for noise cancellation in both active and passive forms. However, in order to fully exploit the possibilities of noise cancellation, we need a formal classification, based on which we can develop noise cancelling circuits systematically.

In short, the basic approach consists of nulling the input ac current which demands knowledge of the noise source (hence sensing of noise source). The nulling operation can be performed with a high-gain active device whose input is applied to the quantity to be nulled, and whose output is applied appropriately to fulfill the circuit condition as restricted by Kirchhoff's laws. Alternatively, passive feedforward can be 


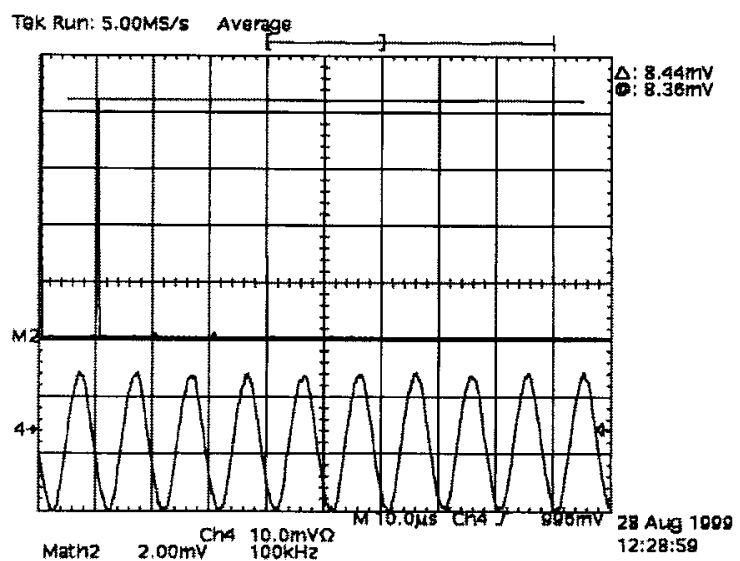

(a)

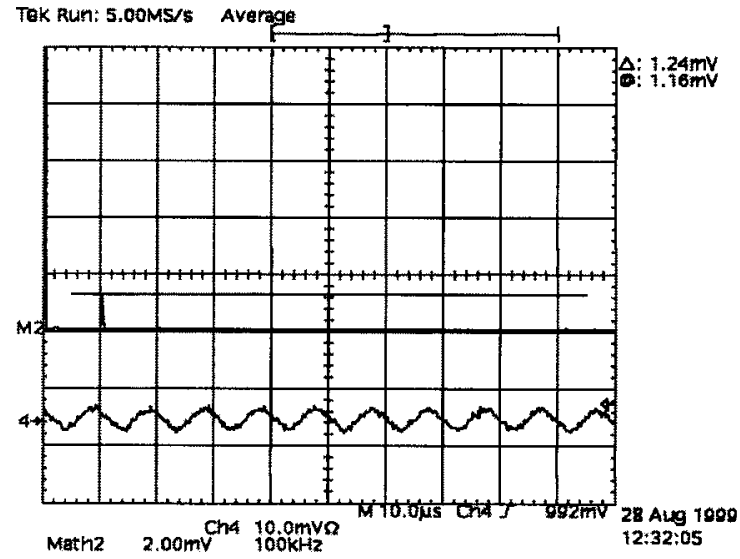

(c)

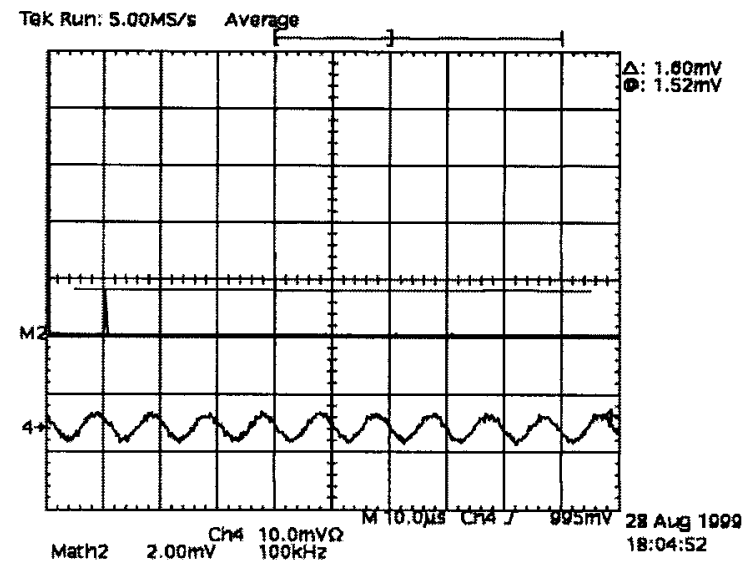

(e)

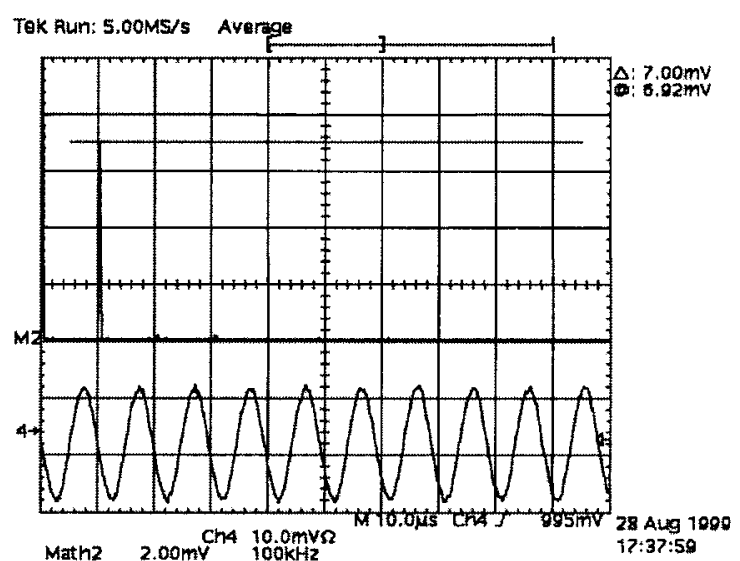

(b)

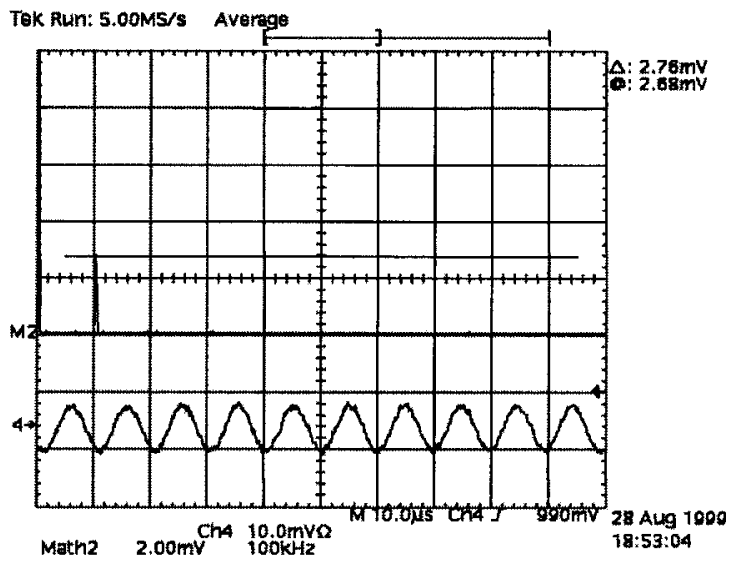

(d)

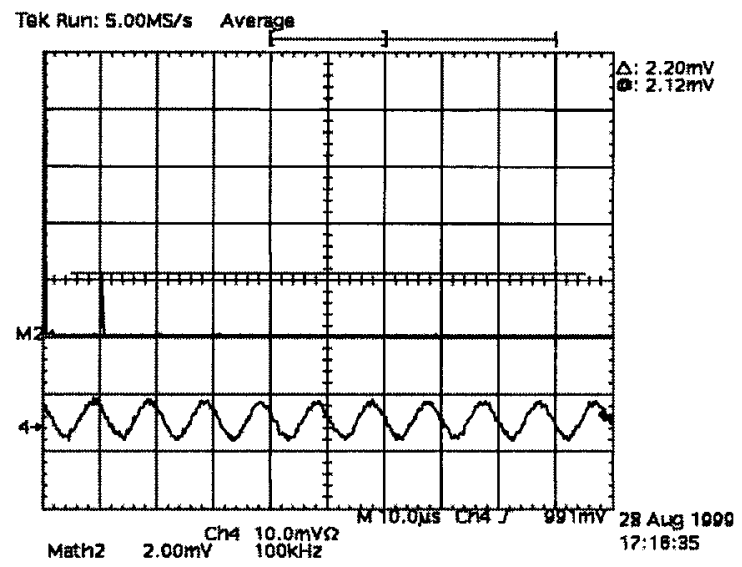

(f)

Fig. 9. Results from experimental circuits of active nullifiers. Upper trace: spectrum (starting $0 \mathrm{~Hz}$, horiz. $100 \mathrm{kHz} /$ div, vert. $4 \mathrm{~mA} /$ div); Lower trace: input current ripple waveform (horiz. $10 \mu \mathrm{s} / \mathrm{div}$, vert. $20 \mathrm{~mA} / \mathrm{div}$ ). (a) Without cancellation. (b) With a $20 \mu \mathrm{H}$ series choke. (c) With active nullifier of Fig. 8a. (d) With active nullifier of Fig. 8b. (e) With active nullifier of Fig. 8c. (f) With active nullifier of Fig. 8d.

applied to achieve "near" null condition. In this paper, we have attempted to classify the basic noise cancellation methods in terms of the basic process of nullification. Specifically, we have derived four fundamental nulling configurations, discussed their practical implementations, and compared their relative merits. Instead of introducing or inventing new circuits, we have focused our attention on the derivation of noise cancelling circuits from first principles.

Practical implementations are numerous. Many papers [3]-[11] were written and patents [13] produced in the past, addressing particular circuit implementations. While "new" circuits continue to be invented, it may be worthwhile to re- 


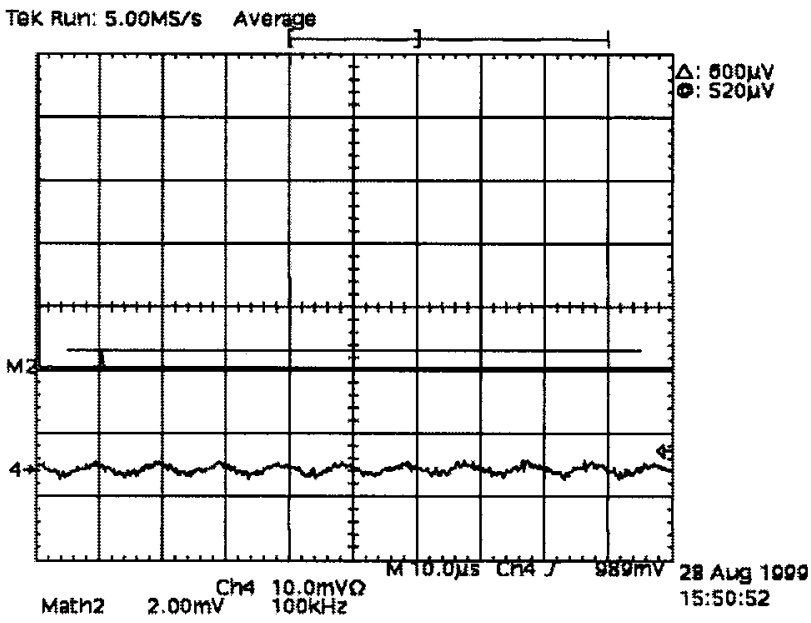

(a)

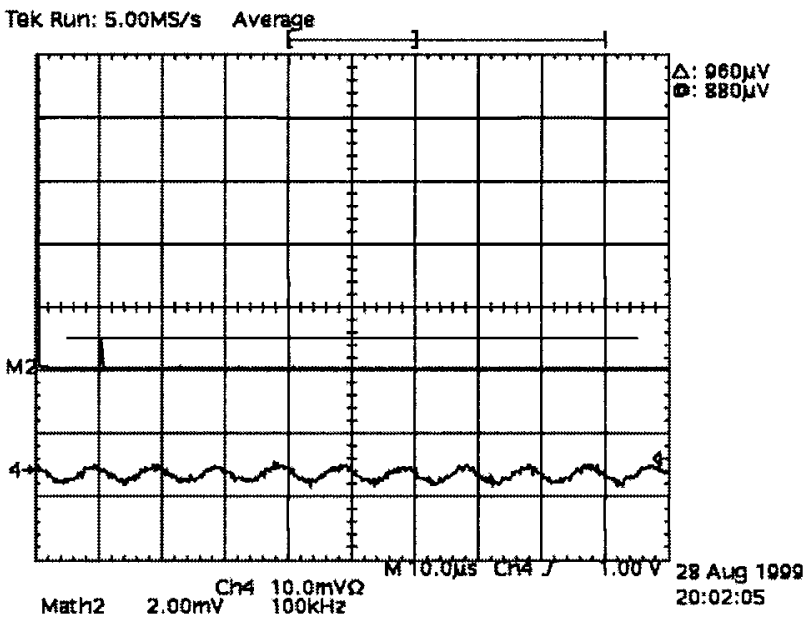

(b)

Fig. 10. Results from experimental circuits of passive nullifiers. Upper trace: spectrum (starting $0 \mathrm{~Hz}$, horiz. $100 \mathrm{kHz} /$ div, vert. $4 \mathrm{~mA} / \mathrm{div}$ ); Lower trace: input current ripple waveform (horiz. $10 \mu \mathrm{s} / \mathrm{div}$, vert. $20 \mathrm{~mA} / \mathrm{div}$ ). (a) With passive nullifier of Fig. 9a. (b) With passive nullifier of Fig. $9 \mathrm{~b}$.

consider the fundamentals in greater depth in order to reinforce the theoretical basis that is necessary for the construction of effective noise cancelling circuits.

\section{ACKNOWLEDGMENT}

The authors would like to thank the anonymous reviewers for their thorough review of this paper and for many constructive comments and suggestions that helped improve the content of this paper.

\section{REFERENCES}

[1] J. Goedbloed, Electromagnetic Compatibility. London, U.K.: Prentice Hall, 1990.

[2] M. J. Nave, Power Line Filter Design for Switched-Mode Power Supplies. New York: Van Nostrand Reinhold, 1991.

[3] T. Farkas and M. F. Schlecht, "Viability of active EMI filters for utility applications," IEEE Trans. Power Electron., vol. 9, pp. 328-337, May 1994

[4] V. Vlatkovic, D. Borojevic, and F. C. Lee, "Input filter design for power factor correction circuits," IEEE Trans. Power Electron., vol. 11, pp. 199-205, Jan. 1996

[5] D. Y. Lee and B. H. Cho, "Design of an input filter for power factor correction (PFC) AC to DC converters employing an active ripple cancellation," in Proc. 3rd Intersoc. Energy Conversion Eng. Conf., 1996, pp. $582-586$.

[6] D. C. Hamill, "An efficient active ripple filter for use in DC-DC conversion," IEEE Trans. Aero. Electron. Syst., vol. 32, pp. 1077-1084, July 1996.

[7] J. W. Kolar, H. Sree, N. Mohan, and F. C. Zach, "Novel aspects of an application of 'zero'-ripple techniques to basic converter topologies," IEEE PESC Rec., pp. 796-803, 1997.

[8] I. Takahashi, A. Ogata, H. Kanazawa, and A. Hiruma, "Active EMI filter for switching noise of high frequency inverters," in Proc. Power Conversion Conf., Nagaoka, Japan, 1997, pp. 331-334.

[9] A. L. Julian, R. Cuzner, G. Oriti, and T. A. Lipo, "Active filtering for common mode conducted EMI reduction in voltage source inverters," in Proc. IEEE APEC, 1998, pp. 934-939.

[10] S. Ogasawara, H. Ayano, and H. Akagi, "An active circuit for cancellation of common-mode voltage generated by a PWM inverter," IEEE Trans. Power Electron., vol. 13, pp. 835-841, Sept. 1998.

[11] D. C. Hamill and P. T. Krein, "A 'zero' ripple technique applicable to any DC converter," in Proc. IEEE PESC Rec., 1999, pp. 1165-1171.

[12] L. Bruton, Active RC Circuits: Theory and Design. Englewood Cliffs, NJ: Prentice Hall, 1980.

[13] J. Marrero and C. Pang, "Ripple current reduction circuit," U.S. Patent 5038263 , Aug. 1991

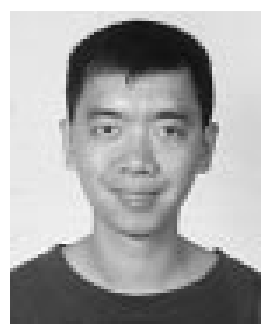

N. K. Poon (M'95) received the B.Eng. degree (with honors) in electronic engineering from the City University of Hong Kong, Kowloon, Hong Kong, in 1995 and is currently pursuing the M.Phil. degree at Hong Kong Polytechnic University, Hong Kong

After graduation, he spent three and a half years with Artesyn Technologies (Asia Pacific), Ltd., and is now with the Power Electronics Laboratory, University of Hong Kong. His current interest includes soft switching techniques, EMI modeling, PFC topologies, synchronous rectification, converter modeling, PWM inverters, and fast transient regulators.

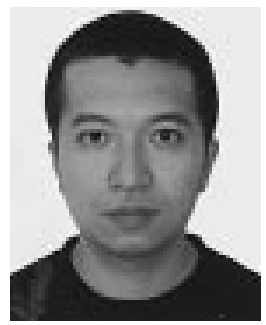

J. C. P. Liu (M'99) was born in Hong Kong. He received the B.S. degree in electrical and electronic engineering from the University of Hong Kong, Hong Kong, in 1993.

After graduation, he joined ASTEC Custom Power, Hong Kong, as an Electronic Engineer, and in 1996 joined Artesyn Technologies (Asia Pacific), Ltd., as a Senior Research Engineer. He is now a Senior Research Engineer with the Power Electronics Laboratory, University of Hong Kong, Hong Kong. His research interest includes high-efficiency synchronous rectification, zero-voltage switching, and converter topologies.

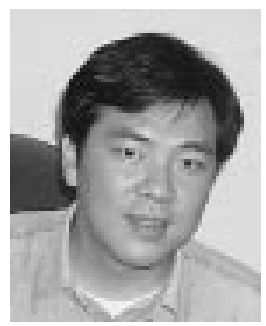

C.K. Tse (M'90-SM'97) received the B.Eng. degree (with honors) in electrical engineering and the Ph.D. degree from the University of Melbourne, Australia, in 1987 and 1991, respectively.

He is a Professor with Hong Kong Polytechnic University, Kowloon, Hong Kong, and his research interests include chaotic dynamics and power electronics. He has worked in software design with an Australian database development company and spent a short period of time with ASTEC Power Modules, Hong Kong, as a Senior Engineer. He is the author of Linear Circuit Analysis (London, U.K.: Addison-Wesley 1998), and co-holder of a U.S. Patent.

Dr. Tse was awarded the L.R. East Prize by the Institution of Engineers, Australia in 1987, the President's Award for Achievements in Research in 1997, and the Excellent Teacher Award, Hong Kong Polytechnic University. He is an Associate Editor for both the IEEE TRANSACTIONS ON POWER ELECTRONICS and the IEEE TRANSACTIONS ON CIRCUITS AND SYSTEMS PART I. 


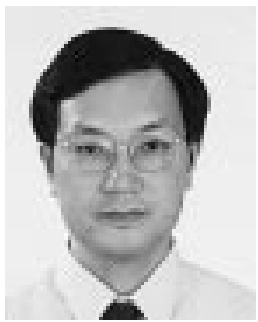

M. H. Pong (M'84-SM'96) was born in Hong Kong. He received the B.Sc. degree in electronic and electrical engineering from the University of Birmingham, U.K., in 1983 and the Ph.D. degree in power electronics from Cambridge University, Cambridge, U.K., in 1987.

After graduation, he became a Senior Design Engineer and then a Chief Design Engineer at National Semiconductor Hong Kong, where he was involved in electronic product design. Afterwards he joined ASTEC International, Hong Kong, first as a Principal Engineer and then a Division Engineering Manager. He is now an Associate Professor with the Department of Electrical and Electronic Engineering, University of Hong Kong, Kowloon, where he is in charge of the Power Electronics Laboratory and leads a team to carry out research in switching power supplies. His research interests include synchronous rectification, EMI issues, power factor correction, magnetic component design, and soft switching. 